\title{
A Unified Field Theory Based on a Rotating de Broglie Wave Packet
}

\section{Louis M. Houston}

The Physics Department, The University of Louisiana at Lafayette, Lafayette, LA, USA

Email: houston@louisiana.edu

How to cite this paper: Houston, L.M. (2019) A Unified Field Theory Based on a Rotating de Broglie Wave Packet. Journal of Applied Mathematics and Physics, 7, 343-355.

https://doi.org/10.4236/jamp.2019.72026

Received: January 18, 2019

Accepted: February 12, 2019

Published: February 15, 2019

Copyright (๑) 2019 by author(s) and Scientific Research Publishing Inc. This work is licensed under the Creative Commons Attribution International License (CC BY 4.0).

http://creativecommons.org/licenses/by/4.0/

\begin{abstract}
We derive a unified field theory based on a rotating de Broglie wave packet that combines electromagnetism, quantum mechanics, gravity and the strong force. We assume the already proven electro-weak force. This Planck units theory requires that $t \approx r$ and $m=r$.

\section{Keywords}

De Broglie Wavelength, Electromagnetism, Quantum Mechanics, Electro-Weak Force, General Relativity, Strong Force
\end{abstract}

\section{Introduction}

Unified field theory, in particle physics is an attempt to describe all fundamental forces and the relationship between elementary particles in terms of a single theoretical framework [1] [2]. In physics, fields that mediate interactions between separate objects can describe forces. In the mid $19^{\text {th }}$ century James Clerk Maxwell formulated the first field theory in his theory of electromagnetism [3]. Then, in the early part of the $20^{\text {th }}$ century, Albert Einstein developed general relativity, a field theory of gravitation [4] [5]. Later, Einstein and others attempted to construct a unified field theory in which electromagnetism and gravity would emerge as different aspects of a single fundamental field [6] [7]. They failed, and to this day gravity remains beyond attempts at a unified field theory.

At subatomic distances, fields are described by quantum field theories, which apply the ideas of quantum mechanics to the fundamental field. In the 1940s quantum electrodynamics (QED), the quantum field theory of electromagnetism, became fully developed [8] [9].

The electroweak interaction is the unified description of two of the four known forces: electromagnetism and the weak interaction [10] [11]. Although these two forces appear very different at everyday low energies, the theory mod- 
els them as two different aspects of the same force.

It is generally believed that a successful grand unified theory (GUT) will still not include gravity. The problem here is that theorists do not yet know how to formulate a workable quantum field theory of gravity based on the exchange of a hypothesized graviton [12] [13] [14]. The current quest for a unified field theory is largely focused on superstring theory and in particular, on an adaptation known as M-theory [15].

One limitation with Einstein's work was at first his rejection, and then his ignorance of quantum theory (which he acknowledged as the years went on). But even today, scientists who are familiar with quantum theory are also having difficulty coming up with a unified theory.

There are many approaches being considered, but the most promising, according to APS, involves string theory. The theory "describes all elementary particles as vibrating strings, with different modes of vibration producing different particles".

In the 1980s, physicists (led by John Schwarz and Michael Green) concluded that string theory could work because of its vibrations. Similar to how a violin plays different notes, the theory went, different vibrations in strings of nature would create different particles. In the mid-1990s, Edward Witten proposed more exact theories of string theory, now called M-theory. His work expanded string theory dimensions from six to seven, and showing new ingredients of the theory (including branes, or membrane-like objects in various dimensions).

Work in this field is very much emerging, but researchers are trying to learn more physical properties of the string by studying the subatomic particles produced in particle accelerators, such as the Large Hadron Collider. Among other directions, the LHC experiments are intended to look for super symmetry, or a supposed mathematical property where every particle species would have a partner particle species.

Physicists caution that a weakness of string theory is it has not yet been confirmed by data. There are also other approaches to unified field theory, such as quantum gravity, which tries to describe gravity in the terms of quantum mathematics.

We have discovered that a de Broglie matter field is a coupled solution to electromagnetism, quantum mechanics, gravity and the strong force. For $\varepsilon$ an extremely small number, the wave packet is considered in the inertial frame of an orbiting mass that is moving at $(c-\varepsilon)$ perpendicular to the orbit and based on the de Broglie condition for integral wavelengths [16] [17]. For these waves, mass and time are set equivalent to radius, allowable because the theory uses Planck units and time is heavily dilated. The "charge" of this field is not that of the electron, but Maxwell's equations are precisely satisfied. In this paper, we present a detailed derivation of the field equations.

\section{Theory}

Given a mass, $m$, moving in a clockwise, circular orbit of constant radius, $r$, at a 
constant velocity, $-v \hat{\theta}$, and moving at $(c-\varepsilon)$ in the positive z-direction, $\varepsilon$ extremely small, there is the de Broglie relationship:

$$
\lambda=\frac{h}{p}
$$

where $\lambda$ is the wavelength of the de Broglie wave, $h$ is Planck's constant [18] and $p$ is the magnitude of the orbital momentum of the mass. The de Broglie orbital condition [19] is:

$$
n \lambda=2 \pi r .
$$

In the inertial frame of the mass, define the following wave fields for the rotating wave packet:

$$
\begin{aligned}
\boldsymbol{B} & =\frac{v}{n} \hat{\theta}, \\
\boldsymbol{E} & =\theta \hat{\theta},
\end{aligned}
$$

with

$$
\theta=\frac{\omega}{n} t
$$

for

$$
\omega=\frac{v}{r} .
$$

In the sections that follow, only the energy due to the rotating fields will be considered.

\subsection{Electromagnetism}

The divergence of $\boldsymbol{B}$ in cylindrical coordinates is:

$$
\nabla * \boldsymbol{B}=\frac{1}{r} \frac{\partial}{\partial r}\left(r B_{r}\right)+\frac{1}{r} \frac{\partial B_{\theta}}{\partial \theta}+\frac{\partial B_{z}}{\partial z}=0 .
$$

The curl of $\boldsymbol{B}$ is:

$$
\nabla \times \boldsymbol{B}=\frac{\hat{r}}{r}\left(\frac{\partial B_{z}}{\partial \theta}-\frac{\partial\left(r B_{\theta}\right)}{\partial z}\right)-\hat{\theta}\left(\frac{\partial B_{z}}{\partial r}-\frac{\partial B_{r}}{\partial z}\right)+\frac{\hat{z}}{r}\left(\frac{\partial\left(r B_{\theta}\right)}{\partial r}-\frac{\partial B_{r}}{\partial \theta}\right)=0 .
$$

The divergence of $\boldsymbol{E}$ is:

$$
\nabla * \boldsymbol{E}=\frac{1}{r} \frac{\partial}{\partial r}\left(r E_{r}\right)+\frac{1}{r} \frac{\partial E_{\theta}}{\partial \theta}+\frac{\partial E_{z}}{\partial z}=\frac{1}{r}
$$

the curl of $\boldsymbol{E}$ is:

$$
\nabla \times \boldsymbol{E}=\frac{\hat{r}}{r}\left(\frac{\partial E_{z}}{\partial \theta}-\frac{\partial\left(r E_{\theta}\right)}{\partial z}\right)-\hat{\theta}\left(\frac{\partial E_{z}}{\partial r}-\frac{\partial E_{r}}{\partial z}\right)+\frac{\hat{z}}{r}\left(\frac{\partial\left(r E_{\theta}\right)}{\partial r}-\frac{\partial E_{r}}{\partial \theta}\right)=0 .
$$

Define the density, $\rho$, with the equation:

$$
\int_{0}^{2 \pi} \operatorname{\rho rd} \theta=1
$$

or 


$$
\rho=\frac{1}{2 \pi r} .
$$

Define the current density, $\boldsymbol{J}$, with the equation:

$$
2 \pi \boldsymbol{J}+\frac{\partial \boldsymbol{E}}{\partial t}=0 .
$$

Based on Equations (4) and (5), (13) becomes:

$$
2 \pi J+\frac{\omega}{n} \hat{\theta}=0
$$

or

$$
\boldsymbol{J}=-\frac{\omega}{2 \pi n} \hat{\theta}
$$

Equations (12) and (14) satisfy the equation of continuity [20]:

$$
\nabla * \boldsymbol{J}+\frac{\partial \rho}{\partial t}=0 .
$$

If we let the permittivity, $\epsilon_{0}$ and the permeability, $\mu_{0}$ be such that:

$$
\varepsilon_{0}=\frac{1}{2 \pi} ; \mu_{0}=2 \pi
$$

(This is explained in the Discussion section.)

So the speed of light is:

$$
c=\frac{1}{\sqrt{\varepsilon_{0} \mu_{0}}}=1 .
$$

Then the system of field equations become:

$$
\begin{gathered}
\nabla * \boldsymbol{E}=\frac{\rho}{\varepsilon_{0}} \nabla * \boldsymbol{B}=0 \\
\nabla \times \boldsymbol{E}=-\frac{\partial \boldsymbol{B}}{\partial t} \nabla \times \boldsymbol{B}=\mu_{0} \boldsymbol{J}+\mu_{0} \varepsilon_{0} \frac{\partial \boldsymbol{E}}{\partial t} .
\end{gathered}
$$

These are Maxwell's equations [21] for an electromagnetic field. Some additional support for these equations is provided by Ampere's law [22]:

$$
\int_{0}^{2 \pi} \boldsymbol{B} * \mathrm{~d} \ell=\mu_{0} I
$$

where $I$ is the enclosed current.

$$
\begin{gathered}
\int_{0}^{2 \pi} \boldsymbol{B} * \mathrm{~d} \ell=\int_{0}^{2 \pi} \frac{v r}{n} \mathrm{~d} \theta=\frac{2 \pi r v}{n}=\lambda v . \\
\int_{0}^{2 \pi} \frac{v r}{n} \mathrm{~d} \theta=\frac{h v}{p}
\end{gathered}
$$

or

$$
\int_{0}^{2 \pi} \operatorname{prd} \theta=n h
$$

Equation (22) is the de Broglie condition. From (20), we see that the enclosed current is: 


$$
I=\frac{r v}{n}
$$

The radiation flux of the electromagnetic field is given by the Poynting vector [23]:

$$
\boldsymbol{S}=\frac{1}{\mu_{0}}(\boldsymbol{E} \times \boldsymbol{B})=0 .
$$

The energy density [24] is:

$$
u=\frac{1}{2}\left(\varepsilon_{0} \boldsymbol{E}^{2}+\frac{1}{\mu_{0}} \boldsymbol{B}^{2}\right) .
$$

The conservation law for the field energy [25] is:

$$
\begin{gathered}
\frac{\partial u}{\partial t}+\nabla * \boldsymbol{S}=-\boldsymbol{J} * \boldsymbol{E} . \\
\nabla * \boldsymbol{S}=0 .
\end{gathered}
$$

Therefore,

$$
\frac{\partial u}{\partial t}=-\boldsymbol{J} * \boldsymbol{E}=\frac{\omega^{2}}{2 \pi n^{2}} t
$$

Equation (28) confirms the current density. The energy circulates through the electric field. Therefore, the total energy, $U_{B}$ stored in the field is due to $\boldsymbol{B}$ :

$$
U_{B}=\frac{\boldsymbol{B}^{2}}{4 \pi \rho}=\frac{1}{2} r \boldsymbol{B}^{2} .
$$

Observe that in this field:

$$
m=r \approx t
$$

This is explained in the Discussion section.

Let the scalar potential be $\varphi$ :

$$
\varphi=-\frac{1}{2} r \theta^{2}
$$

The gradient of $\varphi$ is given as:

$$
\begin{gathered}
\nabla \varphi=\frac{\partial \varphi}{\partial r} \hat{r}+\frac{1}{r} \frac{\partial \varphi}{\partial \theta} \hat{\theta}+\frac{\partial \varphi}{\partial z} \hat{z} . \\
\nabla \varphi=-\theta \hat{\theta}=-\boldsymbol{E} .
\end{gathered}
$$

The vector potential, $\boldsymbol{A}$, is given by the equation:

$$
\boldsymbol{B}=\nabla \times \boldsymbol{A} .
$$

Therefore, we must have:

$$
\boldsymbol{B}=\frac{\hat{r}}{r}\left(\frac{\partial A_{z}}{\partial \theta}-\frac{\partial\left(r A_{\theta}\right)}{\partial z}\right)-\hat{\theta}\left(\frac{\partial A_{z}}{\partial r}-\frac{\partial A_{r}}{\partial z}\right)+\frac{\hat{z}}{r}\left(\frac{\partial\left(r A_{\theta}\right)}{\partial r}-\frac{\partial A_{r}}{\partial \theta}\right) .
$$

Equation (35) reduces to:

$$
\boldsymbol{B}=-\hat{\theta}\left(\frac{\partial A_{z}}{\partial r}-\frac{\partial A_{r}}{\partial z}\right)
$$


Since $r$ is constant, we must have:

$$
\begin{aligned}
\boldsymbol{B} & =\frac{\partial A_{r}}{\partial z} \hat{\theta} . \\
A_{r} & =\int \frac{v}{n} \mathrm{~d} z=\frac{v z}{n},
\end{aligned}
$$

from which we can deduce:

$$
\boldsymbol{A}=\frac{v z}{n} \hat{r} .
$$

Therefore:

$$
\boldsymbol{E}=-\frac{\partial \boldsymbol{A}}{\partial t}-\nabla \varphi
$$

We see that:

$$
\nabla * \boldsymbol{A}=\frac{1}{r} \frac{\partial}{\partial r}\left(r A_{r}\right)+\frac{1}{r} \frac{\partial A_{\theta}}{\partial \theta}+\frac{\partial A_{z}}{\partial z}=0 .
$$

So, this is a Coulomb gauge [26].

\subsection{Quantum Mechanics}

Since the de Broglie wave is a "matter" wave, the quantum mechanical wave function of the de Broglie wave packet is simply the phase wave:

$$
\psi=\sqrt{\rho} \mathrm{e}^{-i E} .
$$

This function is normalized since:

$$
\int_{0}^{2 \pi} \psi^{*} \psi r \mathrm{~d} \theta=1
$$

The traditional Schrodinger equation [27] is:

$$
i \hbar \frac{\partial \psi}{\partial t}=-\frac{\hbar^{2}}{2 m} \nabla^{2} \psi+U \psi
$$

However, for this field, $m=r$. Therefore, the equation becomes:

$$
i \hbar \frac{\partial \psi}{\partial t}=-\frac{\hbar^{2}}{2 r} \nabla^{2} \psi+U \psi
$$

From the de Broglie condition, we get $\hbar=\left(\frac{v}{n}\right) r^{2}$. The energy eigenvalue derives from:

$$
i \hbar \frac{\partial \psi}{\partial t}=\hbar\left(\frac{\omega}{n}\right) \psi
$$

Consequently, the total energy of the system is given by the Hamiltonian, $H$ :

$$
H \psi=\hbar\left(\frac{\omega}{n}\right) \psi=r\left(\frac{v}{n}\right)^{2} \psi
$$

or

$$
\begin{gathered}
H \psi=r \boldsymbol{B}^{2} \psi . \\
\nabla^{2} \psi=\frac{1}{r^{2}} \frac{\partial^{2} \psi}{\partial \theta^{2}}=-\frac{1}{r^{2}} \psi .
\end{gathered}
$$


Therefore,

$$
-\frac{\hbar^{2}}{2 r} \nabla^{2} \psi=\left(\frac{1}{r^{2}}\right)\left(\frac{\left(\frac{v}{n}\right)^{2}}{2} r^{3}\right) \psi=\left(\frac{1}{2} r\left(\frac{v}{n}\right)^{2}\right) \psi
$$

or

$$
-\frac{\hbar^{2}}{2 r} \nabla^{2} \psi=\left(\frac{1}{2} r \boldsymbol{B}^{2}\right) \psi
$$

The potential energy, $U$, is the magnetic energy stored in the field: $U_{B}=\frac{1}{2} r \boldsymbol{B}^{2}$. Therefore:

$$
U \psi=\left(\frac{1}{2} r B^{2}\right) \psi
$$

and the Schrodinger Equation (44) is satisfied.

\subsection{General Relativity}

We can calculate the field tensor [28]:

$$
F^{\alpha \beta}=\partial^{\alpha} A^{\beta}-\partial^{\beta} A^{\alpha},
$$

where $\partial^{\alpha}=\left(\frac{\partial}{\partial x_{0}}-\nabla\right)$ and $\left(x_{0}, x_{1}, x_{2}, x_{3}\right)=(t, r, \theta, z)$. This results in:

$$
F^{\alpha \beta}=\left(\begin{array}{cccc}
0 & -1 / 2 E_{\theta}^{2} & -E_{\theta} & 0 \\
1 / 2 E_{\theta}^{2} & 0 & 0 & B_{\theta} \\
E_{\theta} & 0 & 0 & 0 \\
0 & -B_{\theta} & 0 & 0
\end{array}\right) .
$$

The covariant form of Maxwell's equations is as follows. The inhomogeneous equations are:

$$
\partial_{\alpha} F^{\alpha \beta}=\mu_{0} J^{\beta}
$$

where $J^{\beta}=(\rho, \boldsymbol{J})$ is the four-current. The homogeneous equations are:

$$
\partial^{\alpha} F^{\beta \gamma}+\partial^{\beta} F^{\gamma \alpha}+\partial^{\gamma} F^{\alpha \beta}=0 .
$$

The scalar curvature, $R$, can be written as:

$$
R=\frac{1}{r} .
$$

The contravariant metric tensor [29] is:

$$
g^{\mu \nu}=\left(\begin{array}{cccc}
-1 & 0 & 0 & 0 \\
0 & 1 & 0 & 0 \\
0 & 0 & 1 / r^{2} & 0 \\
0 & 0 & 0 & 1
\end{array}\right)
$$

The relationship between the Ricci curvature tensor [30], $R_{\mu v}$ and the scalar curvature is given by: 


$$
R=g^{\mu v} R_{\mu v} .
$$

The covariant metric tensor is:

$$
g_{\alpha \beta}=\left(\begin{array}{cccc}
-1 & 0 & 0 & 0 \\
0 & 1 & 0 & 0 \\
0 & 0 & r^{2} & 0 \\
0 & 0 & 0 & 1
\end{array}\right) .
$$

The covariant field tensor can be derived from:

$$
F_{\alpha \beta}=g_{\alpha \gamma} F^{\gamma \delta} g_{\delta \beta}
$$

or

$$
F_{\alpha \beta}=\left(\begin{array}{cccc}
0 & 1 / 2 E_{\theta}^{2} & r^{2} E_{\theta} & 0 \\
-1 / 2 E_{\theta}^{2} & 0 & 0 & -B_{\theta} \\
-r^{2} E_{\theta} & 0 & 0 & 0 \\
0 & B_{\theta} & 0 & 0
\end{array}\right) .
$$

The contravariant stress-energy tensor [31], $T^{\alpha \beta}$ can be derived from the following:

$$
T^{\alpha \beta}=-\frac{1}{2 \pi} g^{\alpha \mu} F_{\mu \lambda} \partial^{\beta} A^{\lambda}-g^{\alpha \beta} L
$$

where $L$ is the Lagrangian density:

$$
L=\frac{1}{4 \pi}\left(\boldsymbol{E}^{2}-\boldsymbol{B}^{2}\right)
$$

The covariant form of the stress energy tensor is derived from:

$$
T_{\mu \nu}=T^{\alpha \beta} g_{\alpha \mu} g_{\beta v}
$$

Substitution of the stress tensor (62), the metric tensor (57) and the scalar curvature (55) into the Einstein equations [32] allows one to determine the Ricci curvature tensor, $R_{\mu \nu}$. The Einstein equations are given as:

$$
R_{\mu v}-\frac{1}{2} g_{\mu v} R+g_{\mu v} \Lambda=\frac{8 \pi G}{c^{4}} T_{\mu v}
$$

with $\Lambda$ and $G$ as the cosmological and gravitational constants, respectively. But since we are using Planck units, $G=c=1$ and because the density is one dimensional, the Einstein equations for this system become:

$$
R_{\mu \nu}-\frac{1}{2} g_{\mu \nu} R+g_{\mu \nu} \Lambda=4 \pi T_{\mu \nu} .
$$

\subsection{The Strong Force}

The Yukawa potential [33] for the strong force is given as:

$$
\phi(r)=-\frac{g^{2}}{r} \mathrm{e}^{-r / R}
$$

where, for the rotating de Broglie wave packet, 


$$
g^{2}=\frac{1}{2 \pi \varepsilon_{0}}=1
$$

and $R$ is the range:

$$
R=\frac{\hbar}{m c} .
$$

But $m \rightarrow r$ and $c=1$, so (67) becomes:

$$
R=\frac{\hbar}{r} .
$$

Equations (65) and (68) can be used to derive:

$$
\phi(r)=-\frac{1}{r} \mathrm{e}^{-1 / B} .
$$

However, the Yukawa potential is a screened Coulomb potential and for this system we must use the scalar "electric" potential given in Equation (31). Consequently, the modified Yukawa potential for the rotating de Broglie wave is:

$$
\phi=-\frac{1}{2} r E^{2} \mathrm{e}^{-1 / B} \text {. }
$$

\section{Discussion}

In this Planck units UFT theory, $c=1$. Since $E / B=c$, this implies that $E=B$. This follows from the equivalence: $t \approx r$ (In terms of relativity, $c t$ is the temporal spatial coordinate, but in this case, $c=1$ ).

$$
\begin{gathered}
B=\frac{v}{n} . \\
E=\theta=\frac{\omega}{n} t=\frac{v}{r n} t \rightarrow \frac{v}{n} .
\end{gathered}
$$

Along with $t \approx r$, the equivalence: $m=r$, ensures the proper energy for the ground state: $n=1$. That is, the total energy of the orbiting mass equals the total energy of the rotating field for $n=1$. In addition, $m=r$ makes the quantum mechanics of the field consistent.

$$
\begin{gathered}
\rho=\frac{1}{2 \pi r} . \\
U_{B}=\frac{\boldsymbol{B}^{2}}{4 \pi \rho}=\frac{1}{2} r \frac{v^{2}}{n^{2}} \Leftrightarrow \frac{1}{2} m \frac{v^{2}}{n^{2}} . \\
U_{E}=\frac{\boldsymbol{E}^{2}}{4 \pi \rho}=\frac{1}{2} r \boldsymbol{E}^{2}=\frac{1}{2} r \frac{\omega^{2} t^{2}}{n^{2}}=\frac{1}{2} r \frac{v^{2} t^{2}}{r^{2} n^{2}} \Leftrightarrow \frac{1}{2} m \frac{v^{2}}{n^{2}}
\end{gathered}
$$

Note that in this system, because the density is one dimensional, the "electric" field constant is given as:

$$
k_{e}=\frac{1}{2 \pi \varepsilon_{0}} .
$$

Consequently, unlike typical Planck units, in which $\varepsilon_{0}=1 / 4 \pi$, in this case, $\varepsilon_{0}=1 / 2 \pi$. 
We let the mass orbiting in the $r-\theta$ plane move in the positive $z$-direction at approximately the speed of light $(c-\varepsilon)$. Because of time dilation, $c t$ remains virtually constant and we select $\mathrm{t}$ such that $t \approx r$.

The magnitude of the gravitational field is just the curvature:

$$
g=G \frac{m}{r^{2}} \rightarrow \frac{1}{r} .
$$

So, the divergence of the electric field is the magnitude of the gravitational field:

$$
\nabla * \boldsymbol{E}=g .
$$

In this theory, the reduced Planck's constant becomes:

$$
\hbar=r^{2}\left(\frac{v}{n}\right) .
$$

But in Planck units, $\hbar=1$. Consequently, the total energy is:

$$
E=\hbar\left(\frac{\omega}{n}\right)=\frac{\omega}{n} .
$$

Furthermore, $\hbar=1$ implies:

$$
r=\sqrt{\frac{n}{v}} .
$$

Consequently,

$$
g=\sqrt{B} .
$$

The "charge" density for this theory is 1 wave packet per orbit or:

$$
\rho=\frac{1}{2 \pi r} .
$$

So the "charge" is $q=1$ (i.e. 1 wave packet), so that we can write:

$$
\rho=\frac{q}{2 \pi r} .
$$

But the charge of an electron in Planck units is:

$$
e=\sqrt{\alpha},
$$

where $\alpha$ is the fine structure constant. Clearly, despite the fact that this UFT includes an "electromagnetic" field, it does not actually incorporate electrons.

We can show that $\frac{v}{n}$ is typically extremely small and $r$ and $m$ are typically extremely large. The de Broglie condition is:

$$
n \lambda=2 \pi r .
$$

This becomes:

$$
\begin{gathered}
n \frac{h}{p}=2 \pi r . \\
n h=2 \pi r m v . \\
\hbar=r m \frac{v}{n} .
\end{gathered}
$$


In SI units, we have:

$$
10^{-34} \mathrm{~J} \cdot \mathrm{s}=r m \frac{v}{n}
$$

Let $r=1 \mathrm{~m}$ and $m=1 \mathrm{~kg}$. Thus, without units, we have:

$$
10^{-34}=\frac{v}{n}
$$

In Planck units, $\hbar=1$, so scaling yields:

$$
1=\frac{v}{n} \times 10^{34}
$$

Consequently, in Planck units, we must have:

$$
\frac{v}{n}=10^{-34}
$$

and

$$
r m=10^{34}
$$

And since $m=r$, we have:

$$
r=10^{17} .
$$

We can demonstrate the effects of relativistic speed as follows. Let $v_{z}=c-\varepsilon$, where $\varepsilon$ is a small perturbation. Then we can calculate the time dilation as:

$$
\begin{gathered}
\Delta t^{\prime}=\Delta t / \sqrt{1-\frac{(c-\varepsilon)^{2}}{c^{2}}} . \\
\Delta t^{\prime}=\Delta t / \sqrt{1-\frac{\left(c^{2}-2 \varepsilon c+\varepsilon^{2}\right)}{c^{2}}} .
\end{gathered}
$$

Since $\varepsilon^{2}$ is negligible and we have:

$$
\Delta t^{\prime}=\frac{\Delta t}{\sqrt{\frac{2 \varepsilon}{c}}}=\sqrt{\frac{c}{2 \varepsilon}} \Delta t .
$$

In Planck units, $c=1$. So we have:

$$
\Delta t^{\prime}=\sqrt{\frac{1}{2 \varepsilon}} \times \Delta t .
$$

Mass is augmented by relativity in a similar fashion:

$$
m \rightarrow \sqrt{\frac{1}{2 \varepsilon}} \times m .
$$

And since $m=r$, we have:

$$
r \rightarrow \sqrt{\frac{1}{2 \varepsilon}} \times r .
$$

\section{Conclusions}

As mentioned in the introduction, the weak force combines with the electro- 
magnetic force to become the electro-weak force at high energies. Therefore, the $r-\theta$, planar rotating de Broglie wave packet moving at $(c-\varepsilon)$ in the positive $z$ direction unifies all four forces and quantum mechanics with the stipulation that the theory uses Planck units, with $t \approx r$ and $m=r$.

Future work will be focused on showing that the unified de Broglie field can absorb and radiate gravitons or spin 2 particles.

\section{Conflicts of Interest}

The author has no conflicts of interest with this work.

\section{References}

[1] Nielsen, R.W. (2014) Changing the Paradigm. Applied Mathematics, 5, 1950-1963. https://doi.org/10.4236/am.2014.513188

[2] Duff, M.J. (2011) String and M-Theory: Answering the Critics. Foundations of Physics, 43, 182-200. https://doi.org/10.1007/s10701-011-9618-4

[3] Nave, R. (2013) Electricity and Magnetism. Hyperphysics. Georgia State University, Atlanta, 11-12.

[4] Gupta, S.N. (1957) Einstein's and Other Theories of Gravitation. Reviews of Modern Physics, 29, 334-336. https://doi.org/10.1103/RevModPhys.29.334

[5] Kraichnan, R.H. (1955) Special-Relativistic Derivation of Generally Covariant Gravitation Theory. Physical Review, 98, 1118-1122.

https://doi.org/10.1103/PhysRev.98.1118

[6] Wuensch, D. (2003) The Fifth Dimension: Theodor Kaluza's Ground-Breaking Idea. Annalen der Physik, 12, 519-542. https://doi.org/10.1002/andp.200310025

[7] Gupta, S.N. (1954) Gravitation and Electromagnetism. Physical Review, 96, 1683-1685. https://doi.org/10.1103/PhysRev.96.1683

[8] Feynman, R. (1985) QED: The Strange Theory of Light and Matter. Princeton University Press, Princeton.

[9] Schwinger, J. (1948) Quantum Electrodynamics. I. A Covariant Formulation. Physical Review, 74, 1439-1461. https://doi.org/10.1103/PhysRev.74.1439

[10] Greiner, W. and Muller, B. (2000) Gauge Theory of Weak Interactions. Springer, Berlin/Heidelberg. https://doi.org/10.1007/978-3-662-04211-3

[11] Abers, E.S. and Lee, B.W. (1973) Gauge Theories. Physics Reports, 9, 1-141. https://doi.org/10.1016/0370-1573(73)90027-6

[12] Ahluwalia, D.V. (2002) Interface of Gravitational and Quantum Realms. Modern Physics Letters A, 17, 1135. https://doi.org/10.1142/S021773230200765X

[13] Gupta, S.N. (1962) Recent Developments in General Relativity. Pergamon Press, New York, 251-258.

[14] Sikkema, A.E. and Mann, R.B. (1991) Gravitation and Cosmology in (1+1) Dimensions. Classical and Quantum Gravity, 8, 219-235. https://doi.org/10.1088/0264-9381/8/1/022

[15] Robertson, D.S. (2007) Goedel's Theorem, the Theory of Everything, and the Future of Science and Mathematics. Complexity, 5, 22-27. https://doi.org/10.1002/1099-0526(200005/06)5:5<22::AID-CPLX4>3.0.CO;2-0

[16] Brown, H.R. and Martins, R.deA. (1984) De Broglie's Relativistic Phase Waves and Wave Groups. American Journal of Physics, 52, 1130-1140. 
https://doi.org/10.1119/1.13743

[17] Espinosa, J.M. (1982) Physical Properties of de Broglie's Phase Waves. American Journal of Physics, 50, 357-362. https://doi.org/10.1119/1.12844

[18] Planck, M. (1901) Ueber das Gesetz der Energieverteilungim Normalspectrum. Annals of Physics, 309, 553-583. https://doi.org/10.1002/andp.19013090310

[19] Catillon (2001) A Search for the de Broglie Particle Internal Clock by Means of Electron Channeling. Foundations of Physics, 38, 659-664.

[20] McMahon, D. (2006) Quantum Mechanics Demystified. McGraw Hill, New York.

[21] Rosen, J. (1980) Redundancy and Superfluity for Electromagnetic Fields and Potentials. American Journal of Physics, 48, 1071. https://doi.org/10.1119/1.12289

[22] Owen, G.E. (2003) Electromagnetic TheoryCourie. r-Dover Publications, Mineola, 213.

[23] Kinsler, P.A., Favaro, A. and McCall, M.W. (2009) Four Pointing Theorems. European Journal of Physics, 30, 759-765.

[24] Richter, F., Florian, M. and Henneberger, K. (2008) Poynting's Theorem and Energy Conservation in the Propagation of Light in Bounded Media. Europhysics Letters, 81, 67005. https://doi.org/10.1209/0295-5075/81/67005

[25] Brown, T.M. (1965) Resource Letter EEC-1 on the Evolution of Energy Concepts from Galileo to Helmholtz. American Journal of Physics, 33, 759-765. https://doi.org/10.1119/1.1970980

[26] Adkins, G.S. (1987) Feynman Rules of Coulomb Gauge Qed and the Electron Magnetic Moment. Physical Review D, 36, 1929-1932. https://doi.org/10.1103/PhysRevD.36.1929

[27] Schrodinger, E. (1926) An Undulatory Theory of the Mechanics of Atoms and Molecules. Physical Review, 28, 1049-1070. https://doi.org/10.1103/PhysRev.28.1049

[28] Wheeler, J.A., Misner, C. and Thorne, K.S. (1973) Gravitation. W.H. Freeman \& Co., New York.

[29] Dodson, C.T.J. and Poston, T. (1991) Tensor Geometry, Graduate Texts in Mathematics. 2nd Edition, Springer-Verlag, Berlin, New York, 130.

[30] Besse, A.L. (1987) Einstein Manifolds. Springer, Berlin. https://doi.org/10.1007/978-3-540-74311-8

[31] Jackson, J.D. (1975) Classical Electrodynamics. John Wiley \& Sons, New York, 602.

[32] Einstein, A. (1916) Die Grundlage der allgemelnen Relativitatstheorie. Annalen der Physik, 354, 769. https://doi.org/10.1002/andp.19163540702

[33] Yukawa, H. (1935) On the Interaction of Elementary Particles. Proceedings of the Physico-Mathematical Society of Japan, 17, 48-57. 